\title{
The Exercise Load of Passengers' Postural Control Against Ship Motion Using Human Energy Expenditure
}

\author{
Renon Doine, ${ }^{*}$ "\# Takanori SaKamakI, ${ }^{* *}$ Hiroaki Seta, ${ }^{* * *}$ Masamitsu Iтo, ${ }^{* * *}$ Akihiko Homma, ${ }^{*}$ Yasuhiro FukuI ${ }^{*}$
}

\begin{abstract}
Body motions required for postural control while standing on a marine craft have not yet been characterized despite the popularity of such vehicles. It also remains unclear whether exercise loads for such postural control are greater than those of activities of daily living. The purpose of this study was, therefore, to investigate the kinematic characteristics and exercise load of passengers' postural control against ship motion. We hypothesized that the exercise load of postural control while standing on a marine craft is greater than that while standing on land, which requires more rotations of the joints to maintain upright stability. In this paper, we report passengers' standing postural motion, passengers' exercise load and compared with other exercise loads on land. The measurement system consisted of three orientation sensors, a calorimeter, and a patient monitor. Orientation sensors capable of measuring linear and angular accelerations were placed on the head and waist of each participant, and on the floor of a small marine craft. Human energy expenditure was measured using the calorimeter. In the small marine craft, participants were either sitting or standing. In the laboratory, participants were sitting, standing, or performing step tests at 30, 40 and 50 steps/min. Standing postural motion against ship motion was analyzed by calculating the root mean square (RMS). On the marine craft, standing postural motions of participants were compared with the motions of a vertical standing rod to show the kinematic characteristics of the standing postural motion. Exercise load was calculated by dividing the 5-min mean energy expenditure in each condition by that in the sitting condition. Passengers' standing postural motion on the craft was mainly rotational motion. Passengers' exercise load increased with an increase in floor heave motion. The exercise load during postural control against ship motion was greater than that during quiet standing but was similar to that during stepping on land, with fairly low exercise intensity. We thus found that passengers had an exercise load attributable to postural motion.
\end{abstract}

Keywords: ship motion, passenger, postural control, human energy expenditure, exercise load.

Adv Biomed Eng. 4: pp. 164-169, 2015.

\section{Introduction}

Marine crafts are an important and popular means of transportation, although the effect of ship motion on passengers has not yet been clarified. In previous studies, we investigated human body motion against ship motion [1] and energy expenditure [2, 3]. We were thus interested in measuring the exercise load of passengers' postural control movements.

The size of exercise load can be measured in terms of exercise intensity, exercise volume, rest interval and way of rest [4]. Energy expenditure is an indicator of exercise intensity. In previous studies of passenger postural control, the motion of the passengers' center of gravity was measured in a marine craft $[5,6]$. Wertheim et al. [7] reported the use of a ship motion simulator for the assessment of passenger energy expenditure and heart rate,

This study was presented at the Symposium on Biomedical Engineering 2014, Tokyo, September, 2014.

Received on August 1, 2014; revised on October 20, 2014, December 29, 2014, and April 6, 2015; accepted on May 20, 2015.

* Graduate School of Advanced Science and Technology, Tokyo Denki University, Saitama, Japan.

** Information and Control Engineering Department, National Institute of Technology, Toba College, Mie, Japan.

**** Maritime Technology Department, National Institute of Technology, Toba College, Mie, Japan.

\# Ishizaka, Hatoyama, Hikigun, Saitama 350-0394, Japan.

E-mail: 13udq04@ms.dendai.ac.jp and found that the energy expenditure and heart rate of passengers increased when a pitch-and-roll motion was generated in the simulator. Additionally, Breidahl et al. [8] measured human energy expenditure on two ships, and found that the pitch-and-heeling motion of a ship also affects participant energy expenditure. However, body motions required for postural control while standing on a marine craft have not been characterized. It also remains unclear whether the exercise loads for such postural control are greater than those during activities of daily living on land.

The purpose of this study was, therefore, to investigate the kinematic characteristics and exercise load of passengers' postural control against ship motion. We hypothesized that the exercise load of postural control while standing on a marine craft is greater than that while standing on land, which requires more rotations of the joints to maintain upright stability. In this paper, we report the passengers' standing postural motion, and their exercise load compared with other exercise loads on land. Passengers' standing postural motion was analyzed by measuring the linear and angular accelerations at the head, the waist and at the floor of a small marine craft. Standing postural motion of passengers was compared with the motion of a vertical standing rod to elucidate the characteristics of their standing postural motion. Exercise load was calculated by energy expenditure. Experiments were performed in a small marine craft and in a laboratory on land. In the marine craft, participants were either sitting or standing. In the laboratory, participants were sitting, standing, or performing step tests. The exercise load of passengers in the small craft was compared with that during step tests in the laboratory. 


\section{Methods}

\subsection{Measurement system description}

The measurement system consisted of three orientation sensors (3DM-GX3-25; MicroStrain, Williston, VT, USA), a calorimeter (METAVINE-N; Vine, Nagoya, Japan), a patient monitor (BSM2401, ZS-930P; Nihon Kohden, Tokyo, Japan), and a satellite compass (SC-60; Furuno Electric Co., Ltd., Hyogo, Japan) (Fig. 1). The orientation sensors were placed on the head and waist of each participant, and on the floor of a small marine craft. These sensors measured linear and angular accelerations. The coordinate system for the analysis of linear and angular accelerations is shown in Fig. 2. The marine craft and the passengers experience motions in six degrees of freedom. These motions are referred to as surge (x-axis, longitudinal motion), sway (y-axis, sideways motion), heave (z-axis, vertical motion), roll (rotational motion about the $x$-axis), pitch (rotational motion about the $y$-axis), and yaw (rotational motion about the $\mathrm{z}$-axis) [10]. The frames of the orientation sensors were transformed to a fixed coordinate system using a coordinate transformation [10] to compare the motion of the floor with human body motion. The calorimeter was based on a mixing chamber method [11], and was used to mea-

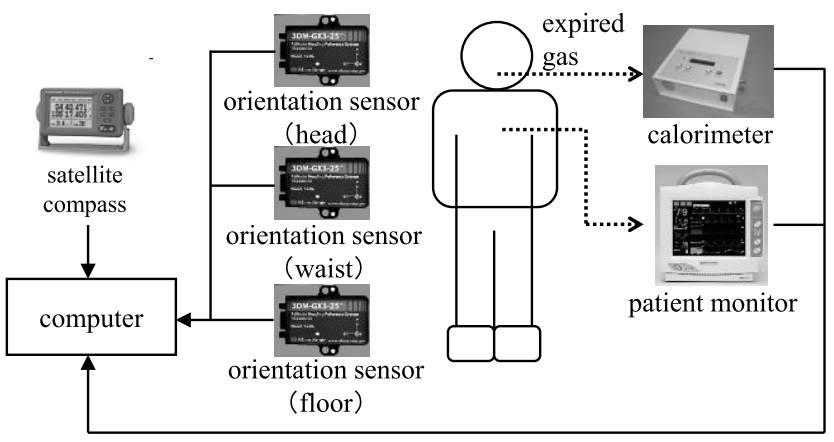

Fig. 1 System diagram.

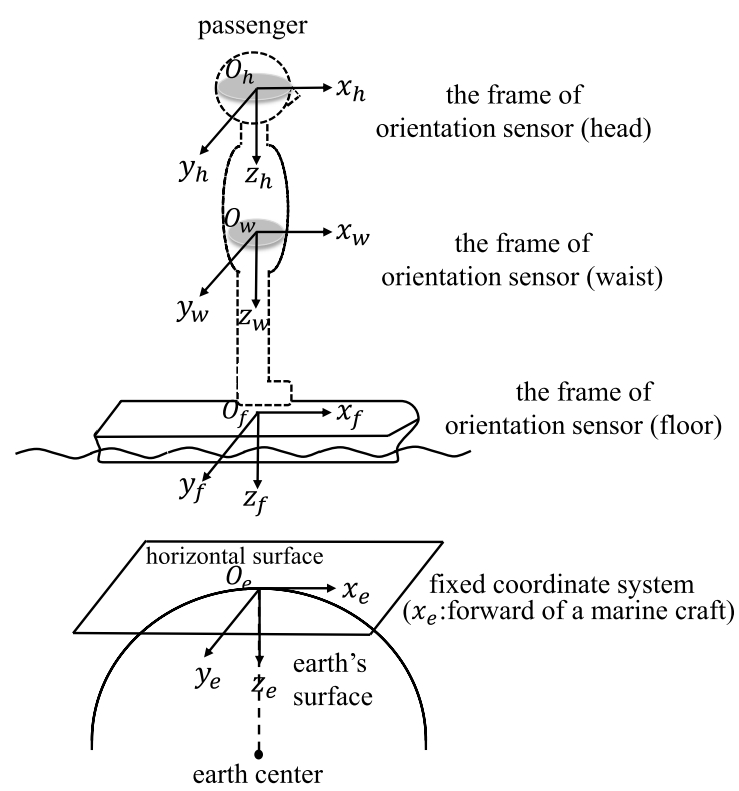

Fig. 2 Coordinate system for analysis of linear and angular acceleration [9]. sure human energy expenditure. The speed and course of the craft were measured using a satellite compass. The sampling intervals were $10 \mathrm{~ms}$ for the orientation sensors, $10 \mathrm{~s}$ for the calorimeter and $1 \mathrm{~s}$ for the satellite compass.

\subsection{Analysis of passengers' postural control 2.2.1 Standing postural motion}

The following parameters were defined to investigate the kinematic characteristics of participants' standing postural motions:

- root mean square (RMS)

- mean RMS

- ratio of mean RMS

The RMS [12] is defined as

RMS $=\sqrt{\frac{1}{N} \sum_{i=0}^{N-1}\left(f_{i}\right)^{2}}$

where $\mathrm{f}$ is linear or angular acceleration, and $\mathrm{N}$ is the number of data points. $\mathrm{N}$ was set at 2048. Linear and angular accelerations at frequencies ranging from 0 to $15 \mathrm{~Hz}$ were observed at the head and the waist. A low-pass filter with a cut-off frequency of $15 \mathrm{~Hz}$ was applied to the linear and angular accelerations to remove noise. Mean RMS was calculated from 15 sequential RMS at the head, waist and floor for each participant. The ratio of the mean RMS at the head or at the waist to the mean RMS at the floor was calculated for each participant. Participants' kinematic characteristics were defined by the average of 18 mean RMS. The 18 mean RMS were chosen according to the mean RMS of floor heave acceleration for 6 participants. Participants' other kinematic characteristics were defined by the average of 18 ratios of mean RMS. The 18 ratios of mean RMS were chosen using the same method used for the mean RMS for the 6 participants. The rod's kinematic characteristics were defined by the average of 5 mean RMS. Participants' kinematic characteristics were compared with the rod's kinematic characteristics. The height of the rod was $160 \mathrm{~cm}$, which was the mean height of the 6 participants.

A signed Wilcoxon rank sum test was performed to examine the differences in linear and angular accelerations among the floor, waist and head. Mann-Whitney's $U$ test was performed to examine the differences in linear and angular accelerations between passengers and the rod. Significance level was set at $\alpha=$ 0.05 .

\subsubsection{Exercise load}

Exercise load was calculated by dividing the 5-min mean energy expenditure in each condition by that in the sitting condition.

In the small marine craft, the participants were either in a sitting or standing posture categorized into three groups based on the mean RMS of floor heave acceleration. In the laboratory, the participants were in a sitting or standing posture, or performing step tests at 30, 40 and 50 steps $/ \mathrm{min}$.

Mann-Whitney's $U$ test was performed to examine the differences in exercise load between the craft and lab settings. Significance level was set at $\alpha=0.05$.

\subsection{Experimental procedure}

Standing postural motion and exercise load were calculated from 3 men and 3 women (age: $20 \pm 2$ years old, height: $160.3 \pm$ $10.6 \mathrm{~cm}$, weight: $62 \pm 18 \mathrm{~kg}$ ). This study protocol was approved by the Bioethics Committee of the National Institute of Technology, Toba College. We obtained informed consent from all study 


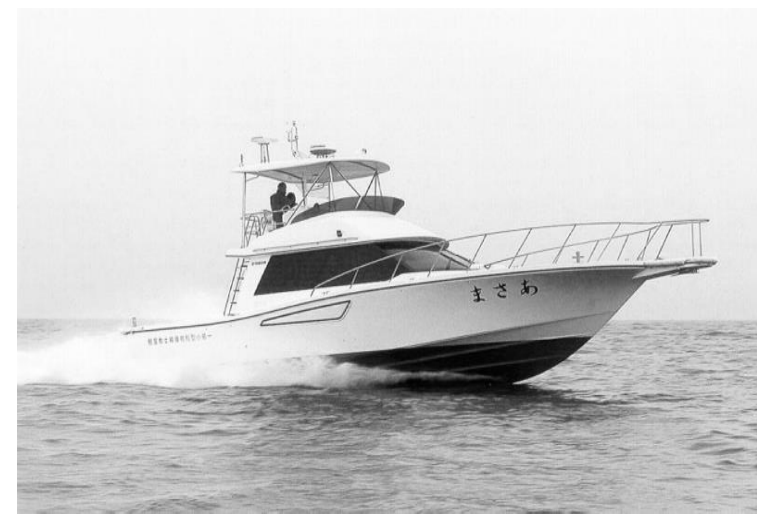

Fig. 3 The small marine craft.

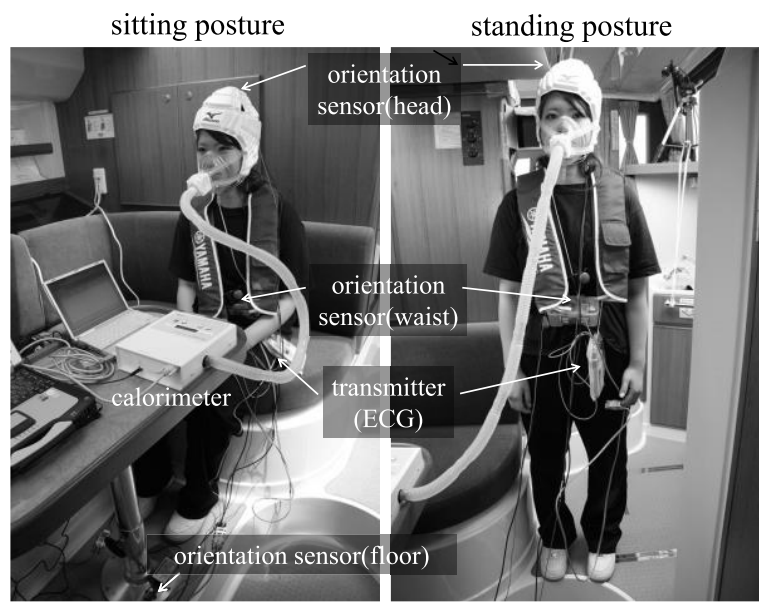

Fig. 4 Experimental set-up in the small craft.
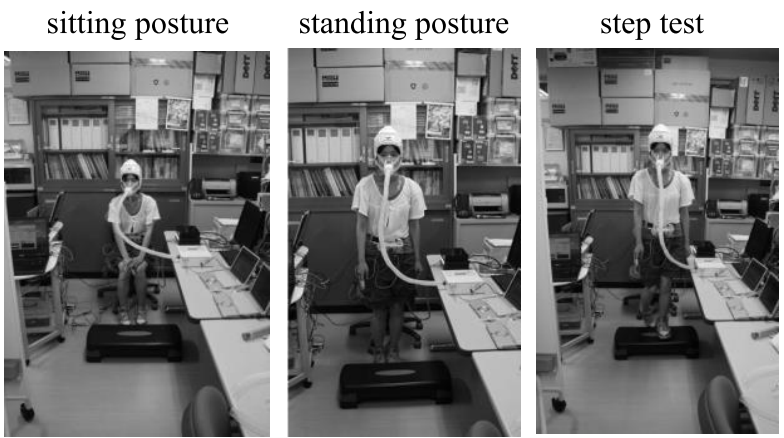

Fig. 5 Experimental set-up in the laboratory.

participants.

The small marine craft and the experimental set-up are shown in Figs. 3 and 4. In the small craft, the experimental protocol was sitting posture for $15 \mathrm{~min}$, standing posture for $15 \mathrm{~min}$, sitting posture for $15 \mathrm{~min}$, standing posture for $15 \mathrm{~min}$, and sitting posture for $15 \mathrm{~min}$. The speed of the small craft was maintained as constant as possible.

The experimental set-up in the laboratory is shown in Fig. 5. In the laboratory, the experimental protocol was sitting posture for $15 \mathrm{~min}$, standing posture for $15 \mathrm{~min}$, sitting posture for $15 \mathrm{~min}$, performing a step test of $30 \mathrm{steps} / \mathrm{min}$ for $5 \mathrm{~min}$, sitting
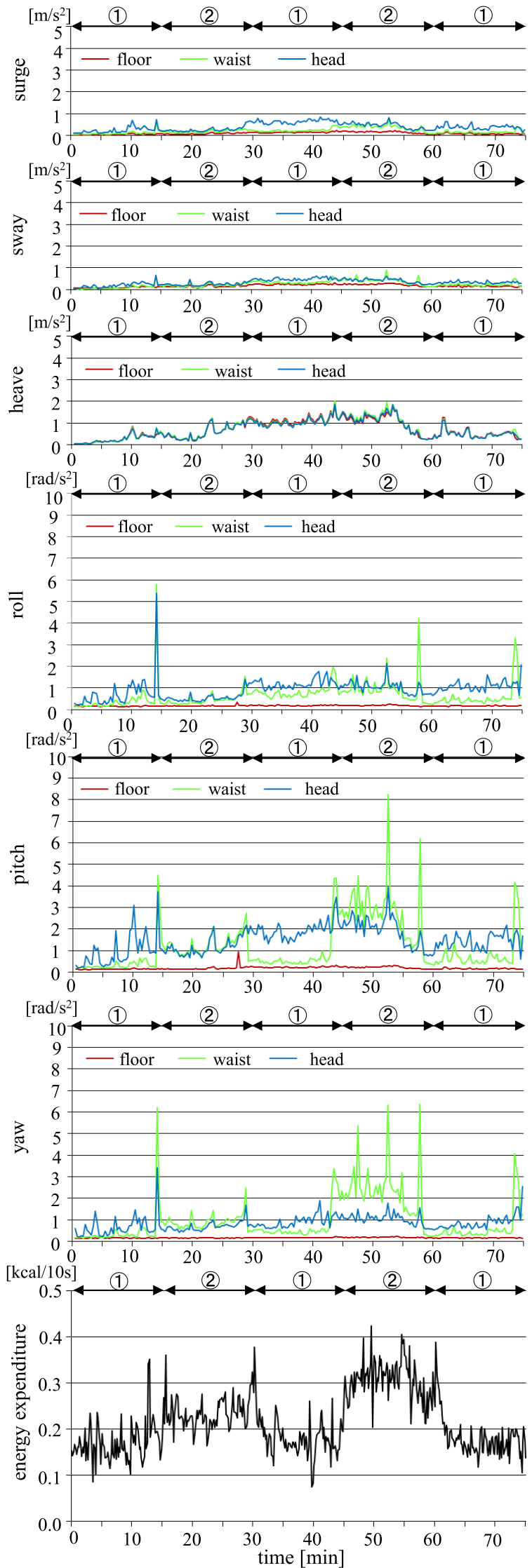

(1)sitting posture, (2)standing posture

Fig. 6 Linear and angular accelerations and energy expenditure from an 18-year-old female participant in the small craft. 
posture for $15 \mathrm{~min}$, performing a step test of $40 \mathrm{steps} / \mathrm{min}$ for $5 \mathrm{~min}$, sitting posture for $15 \mathrm{~min}$, performing a step test of $50 \mathrm{steps} / \mathrm{min}$ for $5 \mathrm{~min}$, and sitting posture for $15 \mathrm{~min}$.

\section{Results}

\subsection{Standing postural motion}

Linear and angular accelerations and energy expenditure from an 18-year-old typical female participant in the small craft are shown in Fig. 6. In this figure, (1) shows the sitting posture and (2) shows the standing posture. In Fig. 6, heave accelerations at the floor, the waist and the head changed simultaneously. Human energy expenditure increased along with the standing postural motion.

Mean and standard deviations of 18 mean RMS of participants are shown in Fig. 7. The mean RMS at the head and the waist were significantly larger than that at the floor $(p<0.05)$. Mean and standard deviations of 18 ratios of mean RMS of participants are shown in Fig. 8. The ratios of angular accelerations at the head and the waist were larger than their corresponding ratios of linear accelerations. Mean and standard deviations of 5 mean RMS of the rod are shown in Fig. 9. Comparing Figs. 7 and 9, the mean RMS of angular accelerations of the rod were smaller than that of the participants $(p<0.05)$.

\subsection{Passengers' exercise load}

Mean and standard deviations of 6 mean RMS categorized into three groups according to the mean RMS of floor heave acceleration are shown in Fig. 10. Postural motions did not change when the mean RMS of floor heave acceleration was less than $1.00 \mathrm{~m} / \mathrm{s}^{2}$.

The mean exercise loads measured in the small craft and in

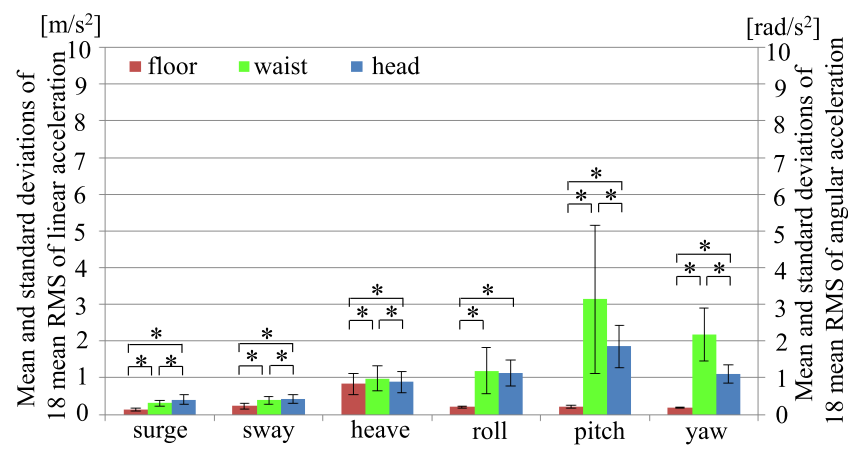

Fig. 7 Mean and standard deviations of 18 mean RMS of participants $\left(\mathrm{n}=18,{ }^{*}: p<0.05\right)$.

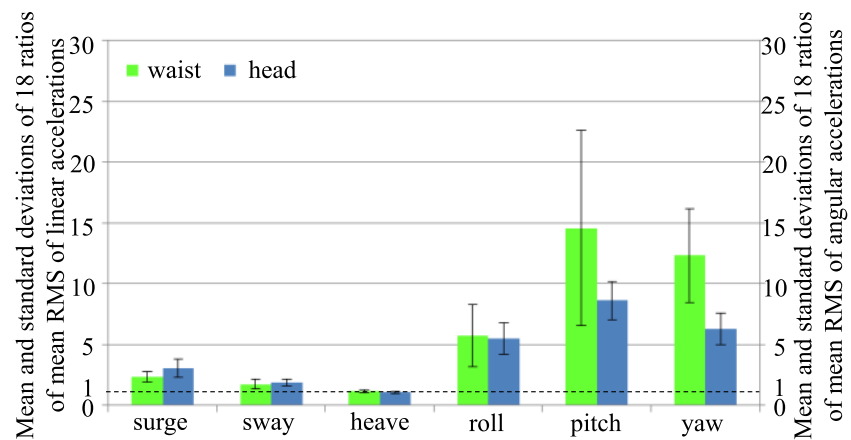

Fig. 8 Mean and standard deviations of 18 ratios of mean RMS of participants $(n=18)$

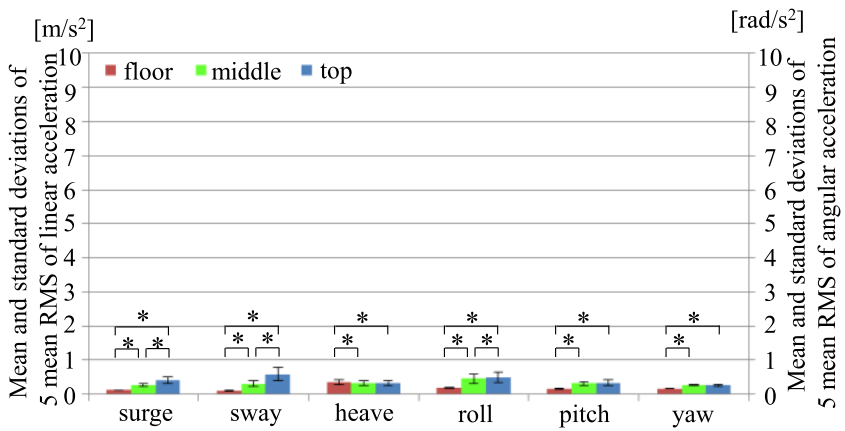

Fig. 9 Mean and standard deviations of 5 mean RMS of the rod $(n=5)$.

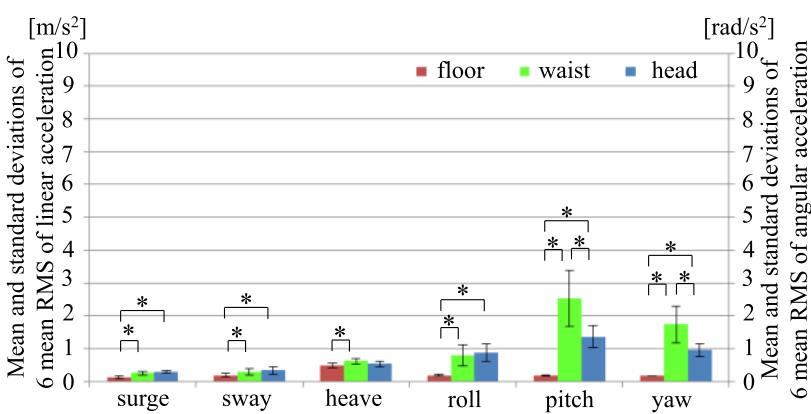

(a) Mean and standard deviations of 6 mean RMS in the $0.20 \mathrm{~m} / \mathrm{s}^{2}$ to $0.59 \mathrm{~m} / \mathrm{s}^{2}$ range of heave acceleration.

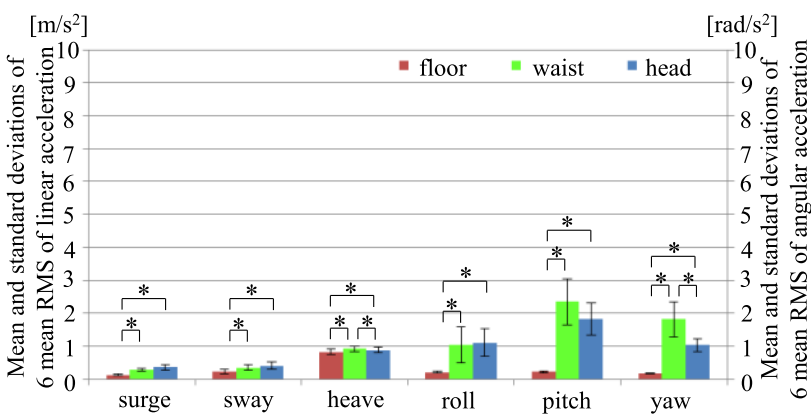

(b) Mean and standard deviations of 6 mean RMS in the $0.60 \mathrm{~m} / \mathrm{s}^{2}$ to $0.99 \mathrm{~m} / \mathrm{s}^{2}$ range of heave acceleration.

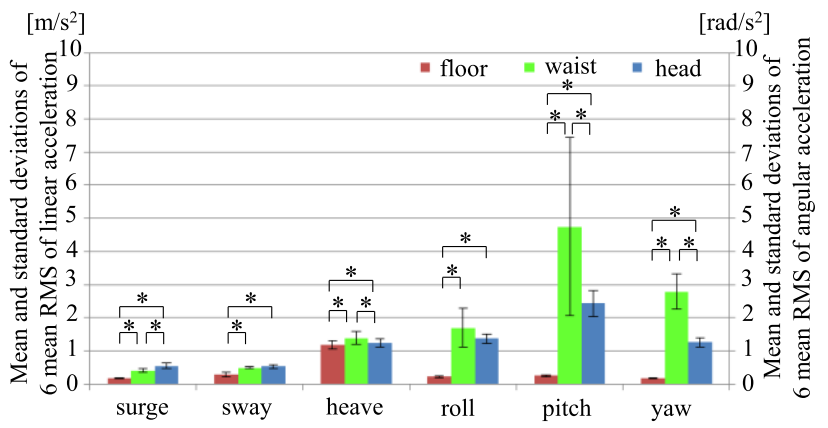

(c) Mean and standard deviations of 6 mean RMS in the $1.00 \mathrm{~m} / \mathrm{s}^{2}$ to $1.39 \mathrm{~m} / \mathrm{s}^{2}$ range of the heave acceleration.

Fig. 10 Mean and standard deviations of 6 mean RMS categorized according to the mean RMS of floor heave acceleration $(\mathrm{n}=$ $\left.6,{ }^{*}: p<0.05\right)$. 


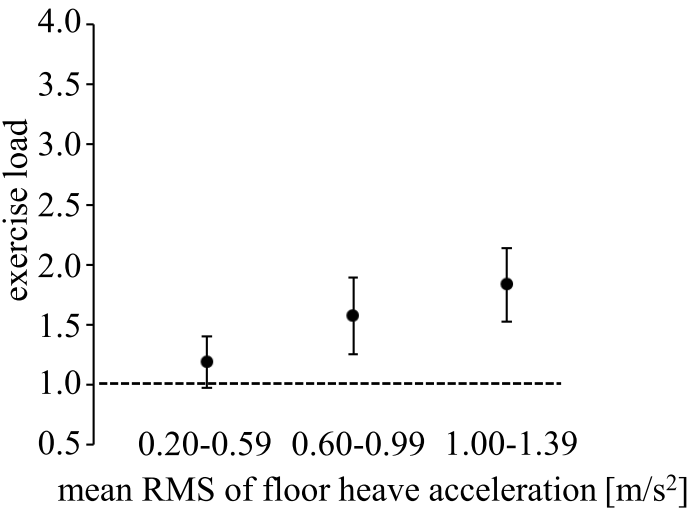

(a) In the small craft

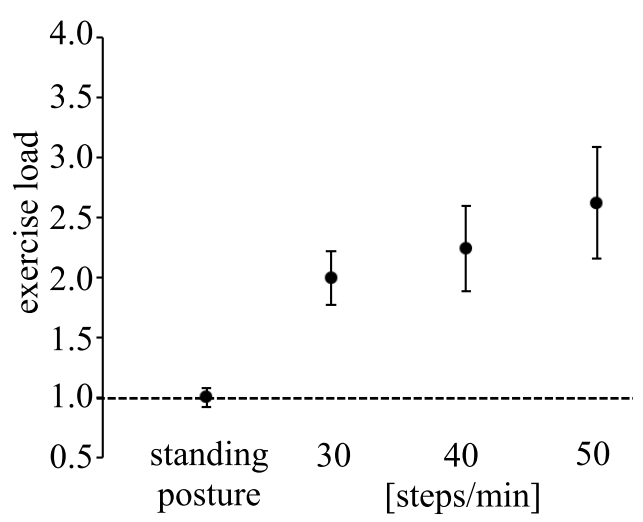

(b) In the laboratory

Fig. 11 Mean exercise loads $(\mathrm{n}=6)$.

the laboratory are shown in Fig. 11. In the small craft, mean exercise load \pm standard deviation were $1.2 \pm 0.2,1.6 \pm 0.3$, and $1.8 \pm$ 0.3 when mean RMS of floor heave acceleration were $0.20-0.59$, $0.60-0.99$ and $1.00-1.39 \mathrm{~m} / \mathrm{s}^{2}$, respectively (Fig. 11a). In the laboratory, mean exercise load \pm standard deviation in the standing posture was $1.0 \pm 0.1$ (Fig. 11b), and were $2.0 \pm 0.2,2.2 \pm 0.4$ and $2.6 \pm 0.5$ during step tests at 30,40 , and 50 steps/min, respectively (Fig. 11b). As shown in Fig. 11a, participants' exercise loads increased with an increase in mean RMS of floor heave acceleration. As shown in Fig. 11b, the exercise load in the standing posture was similar to that when sitting. During step tests, exercise load increased with an increase in intensity of the step test. No significant differences in exercise load were detected between standing on the small craft when mean RMS of floor heave acceleration was $1.00-1.39 \mathrm{~m} / \mathrm{s}^{2}$ and during step tests at 30 or 40 steps/min $(p>0.05)$.

\section{Discussion}

There are three basic strategies for human postural control: the ankle, hip and stepping strategies [13]. Our results suggest that passengers maintain posture by combining these strategies in their own way, depeding on the ship motion. The rotational motions of the head and waist observed in the passengers indicate that they used ankle and hip strategies to maintain an upright posture.

As heave motion increases, more rotations of the ankle and hip joints are required to maintain upright stability, which in- creases exercise load. The exercise load while standing on the craft was found to be greater than that while standing on land, but similar to that during stepping on land. These results suggest that exercise load for postural control against ship motion is greater than that for quiet standing, but is similar to that during stepping on land, with fairly low exercise intensity.

\section{Conclusion}

In this study, we proposed methods of measuring and analyzing passengers' standing postural motions and passengers' exercise load against ship motion. We performed the experiments in a small marine craft and in a laboratory on land.

Passengers demonstrated rotational motions of the head and waist, which would be the result of ankle and hip strategies to maintain upright stability. The exercise load while standing on the marine craft was found to be greater than that while standing on land, but similar to that during stepping on land. These results suggest that exercise load for postural control against ship motion is greater than that for quiet standing but similar to that for stepping on land, with fairly low exercise intensity.

Passengers on marine crafts include people using medical equipment that is not designed for use on such vehicles. The results of this study show that human energy expenditure and passengers' postural motion on a marine craft are different from those on land. The results will thus benefit the research and development of medical equipment for use on a marine craft. For example, the current Holter electrocardiograph has a sensor of linear acceleration which records the patient's activities. This device may need an additional sensor of angular acceleration for use on a marine craft. A demand cardiac pacemaker used on a marine craft must most likely be able to control pacing according to human energy expenditure.

In the shipbuilding field, the International Organization for Standardization has published guidelines for the measurement, reporting and evaluation of vibrations with regard to habitability on passenger and merchant ships (ISO6954) [14]. These guidelines are for the evaluation of vibration based only on ship motion, while passengers' physiological indicator is not considered in the guidelines. The results of the present study allow the development of guidelines for the evaluation of vibrations reflecting passengers' physiological indicator, which may be useful in developing shipbuilding techniques that achieve high habitability.

\section{Conflicts of interest}

We have no conflicts of interest with any companies or commercial organizations based on the definition of the Japanese Society of Medical and Biological Engineering.

\section{Acknowledgment}

This research was supported by JSPS KAKENHI Grant Number 19651075, the Foundation for Applied Research and Technological Uniqueness at N.U.T (Nagaoka University of Technology, 2008), the Sasakawa Scientific Research Grant from The Japan Science Society $(24-724,2012)$, and the National Institute of Technology, Toba College.

\section{References}

1. Doine R, Sakamaki T, Seta H, Ito M, Fukui H, Kawamura T, Fukui Y: Elucidation of the mechanism of the propagation of mo- 
tions and oscillations inside human body caused by a craft. Proc. of the 51st Annual Conference of Japanese Society for Medical and Biological Engineering. 50, pp. 207, 2012.

2. Sakamaki T, Doine R, Seta H, Ito M, Fukui H, Kawamura T, Fukui Y: Fatigue analysis of passengers and crews on a craft. Proc. of the 51st Annual Conference of Japanese Society for Medical and Biological Engineering. 50, pp. 208, 2012.

3. Sakamaki T, Doine R, Seta H, Ito M, Yamada H, Fukui H, Kawamura T, Homma A, Fukui Y: Medical assistance-ship at a time of disaster, and fluctuations of vital sign on a ship. Proc. of the Society of Life Support Engineering (LIFE2013). 61, 2013.

4. Zahradnik D: The Introduction into Sports Training. Masaryk University. pp. 14-31, 2012.

5. Tomonaga S: Postural control in the strange environment. Bulletin of Faculty of Education, Nagasaki University. 57, pp. 107112, 1999.

6. Imaeda Y, Nakahara S, Kimura R, Miki T: Postural movements in standing human induced by ship's rolling and pitching. I. Journal of Japan Institute of Navigation (JIN). 72, pp. 117-124, 1985.

7. Wertheim AH, Heus R, Vrijkotte TGM: Energy expenditure, physical work load and postural control during walking on a moving platform. TNO Defense Research, Soesterberg, 1994.

8. Breidahl T, Christensen M, Jepsen JR, Johansen JP, Omland Ø: The influence of ship movements on the energy expenditure of fishermen: a study during a North Sea voyage in calm weather. Int Marit Health. 3, pp. 114-120, 2013.

9. Doine R, Sakamaki T, Seta H, Ito M, Homma A, Fukui Y: Analysis of the standing postural motion of passengers against ship motion. Life Support. 27(2), pp. 45-53, 2015.

10. Thor IF: Handbook of Marine Craft Hydrodynamics and Motion Control. John Wiley \& Sons, Ltd., Chichester, pp. 5-6, 22-23, 2011.

11. Tamura T, Ichinoseki N, Yoshimura T, Torii Y: Development and evaluation of a simple calorimeter for the measurement of resting metabolism. Clin Exp Pharmacol Physiol. 29, pp. S2-S6, 2002.

12. Edminister JA: Theory and Problems of Electric Circuits, Schaums Outline Series. Schaum Publishing Co., New York, pp. 16, 1965.

13. Horak FB, Nashner LM: Central programming of postural movements: Adaptation to altered support-surface configurations. J Neurophysiol. 55(6), pp. 1369-1381, 1986.

14. International Organization for Standardization: Mechanical vibration - Guidelines for the measurement, reporting and evaluation of vibration with regard to habitability on passenger and merchant ships. Second edition. ISO6954. 2000.

\section{Renon DoINe}

Renon DoINE received her bachelor's degree at the National Institute of Technology, Toba College and her master's degree at Tokyo Denki University in 2013. She has been a doctoral student at the Graduate School of Advanced Science and Technology, Tokyo Denki University. She is a member of the

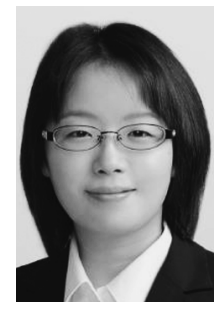
Japanese Society for Medical Biological Engineering, the Japan Society for Precision Engineering, the Japanese Institute of Navigation and the Society of Life Support Engineering.

\section{Takanori SAKAMAKI}

Takanori SAKAMAKI is a professor of National Institute of Technology, Toba College, Japan. He received BS degree at Tokyo Denki University in 1986, MS degree at Tokyo Denki University in 1988, and PhD degree at Tokyo Denki University in 2004. His research field is Medical and Biologi-

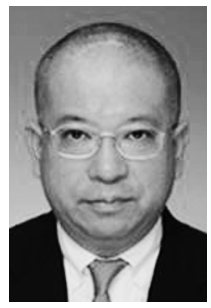
cal Engineering. He is a member of JSMBE, SLSE, JSPE, JIN, JAAP, and IEEE.

\section{Hiroaki SETA}

Hiroaki SETA is an associate professor in maritime technology department at National Institute of Technology, Toba College. He has earned Ph.D. degree in Maritime Science and Technology, at Kobe University in 2005. He holds an unlimited chief mate's license on all ocean-going vessels. His

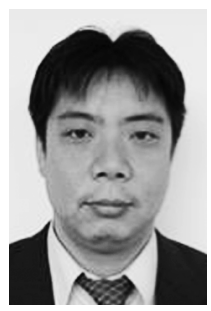
research areas of interest are marine traffic engineering, maritime safety, bridge simulator and maritime education.

\section{Masamitsu Iто}

Masamitsu ITo is a professor emeritus of National Institute of Technology, Toba College. He received his master's degree from Hiroshima University, Japan, in 1976. From 1976 through 1988 he engaged developing marine propeller, car air bag system, dental precision casting, turbopump and light-

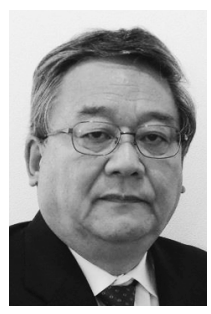
weight fire ladder at Kobe Steel Ltd. From 1988 through 2014 he was a professor of naval architecture at the maritime technology division of Toba College. He is a member of JASNAOE, JSME, JSASS, VSJ and JWEA.

\section{Akihiko Номма}

Akihiko Номма is a professor of the school of science and engineering at Tokyo Denki University, Japan. He finished a doctor's degree of engineering at Tokyo Denki University in 1996. His research field is Medical and Biological Engineering. He is a member of JSAO, JSMBE, SLSE, IEEJ, JSME, IEICE and JSPE.

\section{Yasuhiro FuKUI}

Yasuhiro FukuI received BS degree at Tokyo Institute of Technology in 1967, MS degree at Purdue University (USA) in 1969, and $\mathrm{PhD}$ degree at the University of Wisconsin (USA) in 1972. He has been a professor of the School of Science and Engineering at Tokyo Denki University from 1983 to

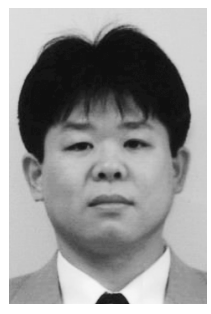
2014. He is a member of Japanese Society for Medical and Biological Engineering, Life support Engineering and Artificial Organs, and the International Society for Rotary Blood Pumps. 